\title{
DAS LEBENSWERK DES FËDOR M. DOSTOJEVSKIJ IM KONTEXT DER EPILEPSIE
}

Gudrun Goes, Magdeburg, Deutschland, gudrun.goes@ovgu.de

Original scientific paper

DOI: 10.31902/fll.38.2021.1

Dichterfreunde und Mediziner neben Selbstausküntften von Dostoevskij bestätigen dessen epileptische Erkrankung. Sie ,begleitete" große Teile seines Lebens, seine Selbstbestimmung und lotete sein dichterisches Schaffen aus. Der Schriftsteller konnte dadurch in Tiefen und Höhen menschlichen Lebens vordringen, u. a. ,steht' Fürst Myškin für diese Ambivalenz. Der Roman avancierte zum Paradigma der Moderne. Medizin und Literatur bilden keine Gegensätze, sondern ihr analytischer Ansatz ist auf den Menschen fokussiert.

Schlüsselwörter: Dostojewskijs Antlitz, Epilepsie: heilige Krankheit, Fluch, Angst oder Transzendenz, Fürst Myškin

\section{Das Bild des Dichters}

Dostojevskij, so Dmitrij Merežkovskij in seinem Dostoevskij-Porträt vom Beginn des 20. Jahrhunderts, habe nie jung ausgesehen:

Mit den Schatten des Leidens und den Falten auf den eingefallenen Wangen, mit der hohen kahlen Stirn, auf der sich die ganze Klarheit und Majestät des Verstandes ausprägt (...), mit dem trüben, wie nach innen gerichteten, unaussprechlich traurigen Blicke der schwach schielenden Augen, den Augen eines Propheten oder Besessenen. (Mereschkowski 180f.)

Dieses Bild des Dichters wurde durch bekannte Porträts, die schon um die Jahrhundertwende zu Dichter-Ikonen geworden waren, überformt. So avancierte das Gemälde von Perov aus dem Jahr 1872 weltweit zum Dostoevskij-Logo. (Vgl. Ljaskovskaja 107-109) Der kontemplative, selbstund weltvergessene Zug, den der Dichter durch seinen abwesenden Blick, die gefalteten Hände und den stark abgedunkelten, das ,Körperlicht' betonenden Hintergrund erhält, hat in Gestik und Mimik auffallende Ähnlichkeit mit Ivan Kramskojs ebenfalls 1872 entstandenem Gemälde ,Christus in der Wüste' und unterstreicht das prophetische Konzept von Perovs Ikonographie. 
Dostoevskijs Witwe Anna Grigor'evna schrieb in ihren Erinnerungen, Vasilij Perov habe im Porträt ihres Mannes „den schöpferischen Augenblick eingefangen, d.h. wie er in sich hineinschaute". (Vgl. Dostojewskaja 248f.) Tatsächlich stellt der Akt der Erfindung, des Entwerfens, Verwerfens und Neukonzipierens von Themen und Konzepten bei Dostoevskij die entscheidende Phase der Textentstehung dar. (Vgl. Catteau 9f.) Dennoch ist die Beschränkung literarischer Arbeit auf den Akt der schöpferischen Eingebung unter Absehung von allen konkreten Arbeitsbedingungen, so wie Maike Schult es festhält, „in Anna Grigorjewnas wie in Perows Darstellung weniger biographisches Faktum als kulturelles Erbe der Genieästhetik, die das mythische Bild des poeta vates, des Dichters als Seher und Zukunftsdeuter, vorgegeben hat". (Schult 111f.) Auf derselben Vorlage beruht der nach einem Foto aus dem Jahre 1878 im Jahr 1895 entstandene Holzschnitt des Schweizer Künstlers Félix Valloton (vgl Ašibaeva 98-101), der später den Schutzumschlag der roten DostoevskijBände des Piper-Verlags schmücken sollte. Über dieses Bild schrieb Thomas Mann: „Es ist auch schrecklich (und rührend bis auf den Grund der Seele!), wieder Dostojewskijs bleiches Heiligen- und Verbrecherantlitz zu betrachten (...)." (Mann 2002, 580)

Die Reduzierung des Dichters auf ein unheimliches Äußeres - hohe Stirn, asketisch eingefallene Wangen und Augen, die vor einem nachtschwarzen Hintergrund in eine schwarze Unendlichkeit zu blicken scheinen - hat jenes entkörperlichte Dostoevskij-Bild maßgeblich befördert, das seit Merežkovskij im Umlauf ist, das von Stefan Zweig gar zur „tragischen Landschaft" verklärt wurde. (Zweig 401) ${ }^{1}$ Wesentlichen Anteil an diesem Stereotyp hat das Verständnis von Dostoevskijs Epilepsie als „heilige Krankheit“. (Mereschkowski 130) Ähnlich wie die Scheinhinrichtung des Dichters vom Dezember 1849 wird damit aus einem Fluch eine Gnade des Schicksals, weil die Krankheit dem Dichter Ausblicke ins Jenseits eröffnet hat: „Die epileptischen Anfälle erscheinen

${ }^{1}$ Vgl. „Rechts und links starren zwei mächtige Steinblöcke, die slawischen Backenknochen heraus, den herben Mund, das brüchige Kinn überwuchert wirrer Busch vom Bart. Erde, Fels und Wald, eine tragische Landschaft, das sind die Tiefen von Dostojewskis Gesicht. (...) Aber wie eine Kuppel weißstrahlend und gewölbt, hebt sich ragend über dem engen bäurischen Gesicht die aufstrebende Rundung der Stirne: aus Schatten und Dunkel steigt blank und gehämmert der geistige Dom: harter Marmor über den weichen Lehm des Fleisches, das wüste Dickicht des Haares. Alles Licht strömt in diesem Antlitz nach oben, (...). Der Körper, der wunde, bäumt sich konvulsivisch in Zuckungen empor (...)." (Zweig 401 und 404) 
Dostojewski wie furchtbare Abgründe, Risse, sich plötzlich auftuende Fenster, durch die er in das jenseitige Licht sehen konnte". (131) In die gleiche Richtung zielt das um den Komplex ,Genie und Krankheit" kreisende Dostoevskij-Bild Thomas Manns, dem auf Seiten Tolstojs „epische Bärenkraft", „ungeheure Naturfrische“ und „heidnische Leiblichkeit" gegenübergestellt werden. (Mann 2009, 43) Dostoevskij dagegen „liebte es [...] nicht, über sich zu reden.“ (Mereschkowski 110) Auch dieses Motiv ist zum festen Bestandteil des Dostoevskij-Mythos geworden, so etwa bei Stefan Zweig: „Schweigsam und scheu war er ein Leben lang. Kaum das Äußerliche, das Körperliche seiner Existenz ist zwingend bezeugt." (Zweig 400) Der Philosoph und Mediziner Josef Rattner sieht die Schwere von Dostoevskijs Schicksal in seiner Gebrechlichkeit; neben seiner Epilepsie habe er an Kopf- und Gliederschmerzen, Malaise mit den Zähnen, Schlafstörungen, an Süchten, an Traumatisierungen durch die bereits erwähnte nicht vollzogene Hinrichtung gelitten. (Vgl. Rattner 43)

\section{Das Leben des Schriftstellers und seine Krankheit, reflektiert in Briefen und Erinnerungen}

Dostoevskijs Vater hatte es als Arzt geschafft, in den Erbadel aufgenommen zu werden. Die Mutter litt unter den seelischen Qualen des ehelichen Zusammenlebens und starb an Schwindsucht zwei Jahre vor dem Tod des despotischen Vaters. Ljubov', die Tochter Dostoevskijs, beschreibt dessen Mutter als eine sanfte, ihrem Mann ergebene Frau, die sich ganz der Familie widmete. Durch acht Geburten war sie erschöpft und musste tagelang im Bett liegen. Solange sie lebte, hielt sie die Familie zusammen. (Vgl. Dostojewskaja 1923, 35-43) Von ihr erbte der Dichtersohn den unverkennbaren Zug der Sentimentalität ${ }^{2}$, die sich bereits in den "Armen Leuten" $(1846)^{3}$ mit dem sozialen Verhalten ${ }^{4}$ verband, um „sein ganzes Werk als tiefes Zeugnis der Menschlichkeit zu prägen." (Onasch 23) Waren die Söhne Michail und Fëdor auch dem Vater äußerlich ähnlich, so liebten sie ihn nicht. Nach dem Tod der Mutter gab sich der Vater vollkommen dem Trunk hin. Er wurde von seinen leibeigenen Bauern erschlagen. Sigmund Freud hat in seinem Aufsatz „Dostojewskij und die Vatertötung" 1928 darauf Bezug genommen. Dieses Verbrechen hinterließ einen tiefen Eindruck auf Dostoevskij und er kam in seinem literarischen Schaffen direkt und indirekt darauf zurück. Seine Tochter schrieb: „Wahrscheinlich war

\footnotetext{
2 sentimentanost'

${ }^{3}$ Bednye ljudi

${ }^{4}$ Social'nost'
} 
Dostojewskij sein ganzes Leben verfolgt von der Vision des blutüberströmten Gespenstes seines Vaters und mit peinlicher Aufmerksamkeit beobachtet er seine Handlungen, immer in der Furcht, er könne die Laster seines Vaters geerbt haben. Dem war nicht so."

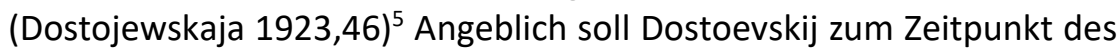
Todes seines Vaters den ersten epileptischen Anfall gehabt haben. Bezeugende Briefwechsel sind zerstört worden. Die Epilepsie oder die Fallsucht wird nach neuesten Erkenntnissen, die aber erst in diachronischer Betrachtung erfahrbar werden, durch plötzliche synchron-oszillierende elektrische Entladung im Gehirn verursacht. Epileptiker können sowohl schreiende Krampfanfälle als auch stumme Anfälle haben. Die Aura ist ein Vorbote eines Anfalls und äußert sich in Kopfschmerzen, Lichtblitzen, Taubheit und Kribbeln in den Extremitäten. Nach einer kurzen Schlafphase können sich Patienten meist nicht an den Anfall erinnern.

In der Erinnerung muss Dostoevskij zwischen Mitleid und Widerwillen gegenüber dem Vater hin- und hergerissen gewesen sein. Freuds Deutungsschema, wonach der Anfall den Wert der Bestrafung habe, weil man einen anderen (meistens Vater oder Mutter) tot gewünscht habe und nun, durch Identifizierung mit diesem Toten, dieser andere werde, versucht, den epileptischen Anfall psychodynamisch zu verstehen. Freud vermutet also eine enge Verbindung von Dostoevskijs eigenen familiären Konflikten, seiner Krankheit und seinem dichterischen Schaffen. (Vgl. Freud 399-418)

So ist zwar Freuds sehr vorsichtig geäußerte Hypothese, Dostoevskij sei von seinen epileptischen Anfällen, die den Sinn einer Selbstbestrafung für den Todeswunsch gegenüber dem gehassten Vater gehabt hätten, im sibirischen Straflager frei geblieben, da ja nun der Zar die unbewusste Strafe vollzogen habe, widerlegt, und zwar durch dessen Briefe, die sein Entsetzen über das vermehrte Auftreten von Anfällen ausdrücken wie auch durch Aussagen von Zeitgenossen. Schon 1845 traf die russische Schriftstellerin Avdot'ja Panaeva Dostoevskij und erinnerte sich an diese Begegnung: „Man konnte auf dem ersten Blick sehen, dass er ein nervöser, eindrucksfähiger junge Mann war, seine Augen blickten unruhig hin und her, und die bleichen Lippen zuckten

\footnotetext{
${ }^{5}$ Insgesamt ist der Wahrheitsgehalt der Aussagen der Tochter über das Leben der Familie zu relativieren und auch einzuschränken, da eine kritische Analyse vermieden wird und sehr einseitig die Beziehungen dargestellt werden. Ihrer Mutter bescheinigt sie eine zu geringe Einbildungskraft. Die Eltern hätten statt Liebe nur Sympathie füreinander empfunden. Einige Details erhellen trotzdem bestimmte Zusammenhänge. (Vgl. Dostojewskaja 1923, 46)
} 
nervös." (Panaeva 156f) In den Briefen an seinen Bruder Michail und überhaupt in seiner gesamten Korrespondenz reflektiert Fëdor seine gesundheitliche Befindlichkeit. So schreibt er im Februar 1847 an seinen Bruder:

Das $\ddot{A}$ ßere muß mit dem Innern im Gleichgewicht sein. Anderenfalls gewinnt bei fehlenden Einwirkungen von außen das Innere auf gefährliche Weise die Oberhand. Die Nerven und die Phantasie nehmen einen großen Raum im Wesen ein. Jede äußere Einwirkung erscheint einem kolossal, wenn man sie nicht gewöhnt ist, und irgendwie erschreckend. Man beginnt sich vor dem Leben zu fürchten" (Dostojewski 1984, 77)

und aus der Peter-Pauls-Festung im August 1849:

(...) nachts werde ich von langen, abscheulichen Träumen geplagt, und obendrein scheint mir seit kurzer Zeit der Boden unter den Füßen zu schwanken (...). Aus all dem schließe ich auf die Zerrüttung meiner Nerven. Habe ich mich früher unter einer solchen Nervenanspannung befunden, so nutzte ich die Zeit zum Schreiben - in der Regel kann man in diesem Zustand besser und mehr schreiben, aber jetzt halte ich mich zurück, um mich nicht endgültig zugrunde zu richten. (85)

Hier wird bereits deutlich, dass diese besondere Nervenanspannung letztlich Dostoevskij schöpferisch beflügelt. Jahre später aus Omsk an Natal'ja Fonvizina, die Frau des Dekabristen Ivan Fonvizin schreibend, wird dieser Gedanke bestätigt:

Ich bin ständiger Erwartung; es scheint, als sei ich noch immer krank und als müsse bald, sehr bald irgend etwas Entscheidendes mit mir geschehen, als komme ich an den kritischen Punkt meines ganzen Lebens, als sei ich gleichsam reif geworden für etwas und als würde etwas vielleicht Leises und Klares, vielleicht Schreckliches, auf jeden Fall aber Unabwendbares kommen. Sonst wäre mein Leben verfehlt. Doch vielleicht entspringt das alles nur meiner kranken Phantasie. (113)

Nach seiner Heirat mit Marija Dmitrievna Issaevna bestätigte ihm ein Arzt in Barnaul 1857, dass er an Fallsucht leide: „(...) und ich müßte damit rechnen, bei einem dieser Anfälle an einem Spasmus in der Kehle zu ersticken, und würde nur so und nicht anders sterben. (217)

Rene Fülöp-Miller hebt in seiner Schrift „Die Krisis in Dostojewskis Leben. Eine Darstellung auf Grund des bisher unbekannt gebliebenen Nachlasses" hervor: 
(...) auch Suworin hat in einem Artikel in der Nowoje Wremja schon 1881 behauptet, daß sich in Dostojewskis Kindheit ,etwas Furchtbares, Unvergeßliches und Qualvolles' ereignet habe, welche Bemerkung auch durch Mitteilungen des Diplomaten und Schriftstellers, Eugène Melchior, Vicomte de Vogůé, des Verfassers von ,Le roman russe', bestätigt zu werden scheine. Und Dr. Janowski, Dostojewskis Arzt und Freund, teilte nach dem Tod des Dichters in einem Schreiben an Anna Grigorjewna gleichfalls mit, Dostojewski habe schon in seiner Kindheit jene düsteren Eindrücke in sich aufgenommen, die dann später zu seiner Nervenerkrankung, wie auch zu jener argwöhnischen Charakterlage bei ihm geführt hatten. (Fülöp-Miller XLV)

Die erbliche Bedingtheit seines Leidens ist durch den Tod seines Sohnes Leša erwiesen, der im Alter von 3 Jahren nach einem epileptischen Anfall starb.

Vermutet wird von Forschern nach Freud, dass die abnorme Triebabfuhr, die sich im epileptischen Anfall äußert, mit schweren, verdrängten Aggressionen und Todeswünschen zusammenhängt. Jung hat 1924 in "Psychologie und Erziehung" mit Hinweis auf Gehör- und Gesichtshalluzinationen diese als Vorstadien der Epilepsie beschrieben. Er berichtet darin von einem Jungen, der mit 7 Jahren anfing, eigentümlich zu werden. Er versteckte sich im Keller, lief weg, gelegentlich hatte er Wutanfälle, bei denen er einmal fast seine Schwester getötet hätte. Er wurde als böses Kind behandelt, bis er mit 12 Jahren den ersten epileptischen Anfall hatte. Jung konnte im Gespräch mit dem Jungen nach längerer Zeit herausbekommen, dass der Junge vor einem kleinen Mann mit Bart Angst hatte. Vor ihm lief er weg. Dieser Mann wollte ihm etwas Schreckliches übergeben. Später nahm er die Schuld an, es war nämlich der Mord, den er beinahe an seiner Schwester begangen hatte. Der schreckliche Mann tauchte nicht wieder auf, dafür aber die Gestalt einer Nonne. Die Wutanfälle hörten trotz wachsender Erregbarkeit auf, stattdessen begannen die manifesten epileptischen Anfälle. (Vgl. Jung 17-20). Im Tagebuch eines Schriftstellers hat Dostoevskij ein ihn sein ganzes Leben lang begleitendes Erlebnis niedergeschrieben: Eine plötzliche GehörHalluzination ließ inn vor Schrecken erstarren, er glaubte, jemanden rufen zu hören: Ein Wolf, ein Wolf. Vor Angst lief er schreiend aufs Feld, wo ein Leibeigener seines Vaters pflügte. Dieser unterbrach seine Arbeit und sah in das zuckende, angstverzerrte Gesicht des Jungen. Er liebkoste seine Wangen und berührte die zitternden Lippen und sagte: Da sei doch gar kein Wolf. (Vgl. Dostojewski 1992, 143f.) Man kann hier vermuten, 
dass sich von dem Jungen Fëdor, ähnlich wie das von Jung beschriebene Beispiel, eine Teilpersönlichkeit abgespalten hatte, von der er in der erschreckenden Halluzination des Wolfes verfolgt wurde.

Anfang der 1860er Jahre war Fëdor Dostoevskij mit den Schwestern Anna Korvin-Krukovskaja und Sof'ja, der später berühmt gewordenen Mathematikerin, befreundet. Auch ihnen gegenüber thematisierte er seine epileptischen Anfälle:

In diesem Augenblick erklang von der nahen Kirche die Glocke der Osterfrühmesse. Die Luft geriet in Schwingung und tönte dumpf. Und ich fühlte, erzählte Fjodor Michailowitsch, wie der Himmel zur Erde kam und mich verschlang. Ich fand wirklich Gott und ward von ihm erfüllt. Ja, Gott ist, schrie ich- und sonst erinnere ich mich an nichts! Ihr seid alle gesunde Menschen, fuhr er fort, und ihr habt nicht die geringste Vorstellung von jenem Glück, das wir Epileptiker in der Sekunde vor dem Anfall empfinden. Mohammed versichert im Koran, dass er das Paradies gesehen habe und dort gewesen sei. Alle klugen Toren sind davon überzeugt, dass er einfach ein Lügner und Betrüger ist. Aber nein! Er lügt nicht! Er war tatsächlich im Paradies, während des Anfalls der Epilepsie, an der er gleich mir litt. Ich weiß nicht, ob diese Glückseligkeit Sekunden oder Stunden oder Monate währt, aber glauben Sie mir aufs Wort, alle Freuden, die das Leben geben kann, würde ich für sie nicht eintauschen! (Kowaleskaja 108)

Dieses hier vom Dichter geschilderte Erlebnis, die Hochstimmung vor dem Anfall, die Transendenz bedeutete, das Erleben des Paradieses, verweisen sehr direkt auf die Reden des 7. Engels aus der JohannesOffenbarung. Und der Leser erlebt dies in der fast gleichen Diktion in den Romanen „Der Idiot“ (1868) . und „Die Dämonen“ (1871)7. Als Dostojevskij sich dem vierzigsten Lebensjahr näherte, nahm seine Fallsucht unerträgliche Formen an, seine junge Frau Anna musste diese Erfahrung immer wieder machen: Sie erwähnt in ihrem Tagebuch seine Krankheit:

( (...) $)^{8}$ ich wurde von Fedja geweckt, weil sein Anfall begann. (...) Ich hätte lieber eine schwere Krankheit durchgestanden als diese unglückliche Lage.), (Dostojewskaja 1976,104 und 214) seine Zornesausbrüche (,Fedja aber begann zu schreien (...), und zwar so

\footnotetext{
${ }^{6}$ Idiot

${ }^{7}$ Besy

8 Vgl. auch Tagebucheintragungen vom: 2.7.1867; 24.5.1867; 18.7.1867;

2.8.1867; 26.9.1867; 27.9.1867, 26.10 1867, 6.11. 1867.
} 
fürchterlich und hemmungslos, (Canaille, gemeines Stück, Aasi) “(373) ${ }^{9}$ und sie beschreibt ihre Träume. Sie glaubte, dass, wo sie auch immer hinkamen, plötzlich das Wetter umschlug oder die Musik zu spielen aufhörte. " (119)

1867 schrieb Dostoevskij seiner ehemaligen Geliebten Polina Suslova, dass sich seine Epilepsie bis zur Missgestalt steigere. (Vgl. Dostojewskij 1968, 212) Nicht nur das seelische Gleichgewicht geriet aus der Balance, auch seine Körperlichkeit veränderte sich zusehends.

Dem Historiker Vs. Solov'ev schildert er 1872 bei einer Begegnung, dass ihn die Krankheit nicht mehr verlasse. (Vgl. Solov'ev 37f) Im Briefwechsel des Dichters mit seinem Bruder, seiner Frau und auch Freunden wird weiterhin seine Krankheit thematisiert, es fallen immer wieder die Worte Nerven, Anfälle, Hochgefühl und Niedergeschlagenheit, Zweifel und Hoffnung. In den ausführlichen Darstellungen seiner Krankheit sieht der Psychologe Paul Vogel, die Möglichkeit, „den pathischen Aspekt der Erkrankung in ganz ungewöhnlicher Weise zu erhellen " (Vogel 31)

In der englischen und amerikanischen Forschung spielt dieses Thema ebenso eine große Rolle. Der Slavist Paul Fung versteht das Schreiben von Dostoevskij als epileptisch und „it is punctuated by moments of caesuras and breaks". (Fung 1) Ihn interessieren weniger die Krankheit des Dichters im Suchen seiner Helden, sondern Brüche und Schweigen im Text, er untersucht das metaphorische Schreiben, die „epileptische“ Narration.

Dostoevskijs Schaffen, das ist besonders hervorzuheben, steht in dem hier skizzierten Zusammenhang sowohl im Fokus der Medizin als auch der Literaturwissenschaft, wobei sich Fragen, Interesse und Zugänge unterscheiden. Unser Interesse liegt in der Aufdeckung der Kausalität von Krankheit und Kranksein und Sinnsuche des Helden, wobei die erzählte Krankheit in literarischen Texten auch eine Eigendynamik entwickeln kann. Während sich die Medizin um die Wirkungsursache bemüht, fragt die Literatur, hier der Schriftsteller, nach dem Zweck; welche Rolle spielen die Helden als Epileptiker? Aber die Kenntnisse der Medizin sind Teil dieser Diskussion, es geht dabeijedoch nicht um die Verifizierung medizinischer Diagnosen mit den literarischen.

9 Die Klammerbemerkung wurde im Original gestrichen. Vgl. auch Tagebucheintragung. vom 1.9.1867. 


\section{Der Schriftsteller F. Dostojewskij und sein Werk: Ist die Epilepsie eine heilige Krankheit?}

Dostojewskij war in Petersburg zum Schriftsteller geworden, im sibirischen Straflager geistig gereift und verändert worden, hier deutet sich an, wie groß Makro- und Mikrokosmos von Dostoevskijs Leben und Werk sind.

Die ungesunden Lebensbedingungen Peterburgs produzierten im 19. Jahrhundert pathologische Verhaltensweisen, die von einem idealen Lebensentwurf eklatant abwichen. Die Stadt als Lazarett war Spiegel und Quelle ungesunder Lebensführung, die Krankheit, Langeweile und Leere hervorbrachte. Im Fokus dieses pathologischen Blickwinkels wurden auch die Gegensätze zwischen arm und reich sichtbar. Dabei ging es dem Dichter nicht um das Nachzeichnen der psychischen Entwicklung als vielmehr um kollektive Verhaltensweisen, deren Ethik dann an den medizinischen Kriterien von gesund und krank gemessen wurden. Es waren falsche Werte einer pragmatischen Gesellschaft. (Vgl. Merten 78ff) Denn das Kranksein konnte keineswegs mit der Abnahme ethischer Anerkennung gleichgesetzt werden.

Die heilige Krankheit hatte eine hohe Dignität. Der antike Mensch sah in der Krankheit die Wirkung eines Göttlichen, welches daher wiederum nur durch ein Göttliches oder einen Gott geheilt werden konnte. Keine andere Krankheit repräsentiert das ganzheitliche oder leibliche, seelische Leiden im Horizont philosophischer oder theologischer Dimensionen mehr als die Epilepsie, meint der Medizinhistoriker Dietrich von Engelhart. Epilepsie wird als Anfallsleiden und als Wesensveränderung angesehen. (Vgl. Engelhardt 25-40) ${ }^{10}$

10 Eine andere Sicht wird durch Prof. Janz vertreten: „Was man in vorhippokratischer Zeit unter, heiliger Krankheit' verstanden hat, können wir nicht mehr direkt erfahren, sondern nur indirekt ermitteln, indem wir die hippokratische These, Epilepsie sei eine natürliche Krankheit, als dialektische Aussage annehmen oder indem wir der Tradition nachgehen, in der sich die Bedeutung dieses Begriffs bis in unsere Zeit erhalten hat. ,Heilig' bedeutet in diesem Zusammenhang sicher keine Auszeichnung, wie man im 17. Jahrhundert irrtümlich gemeint hat, als man dieses Epitheton einer besonderen Intelligenz, Genialität oder gar einem prophetischen Vermögen zuschrieb, sondern eindeutig ein Verruf. Auch lag es antikem Bewußtsein gänzlich fern, Epileptiker als heilige und Propheten zu verehren. İ́pòs und sacer gehören zu den Urworten, die (...) einen Doppelsinn haben, der ebenso heilig wie verflucht oder beides bedeuten konnte." (Janz 1966, 2f.) 
Der Dichter geht sehr frei mit der Selbsterfahrung seiner Epilepsie um, das hat sein Briefwechsel nachhaltig bezeugt. Er hat sein ganzes Selbst in sein Werk gelegt, schreibt Nikolaj Berdjaev:

Und an Hand seines Werkes kann man ihn studieren. Darum ist Dostojewskij weniger rätselhaft als viele andere Schriftsteller. (...) Er hat uns sein „sodomitisches" Ideal nicht verhehlt und die Höhen seines „Madonnen“-Ideals erschlossen. Darum ist das Schaffen Dostojewskijs eine Offenbarung. Die Epilepsie ist keine oberflächliche Krankheit, in ihr offenbaren sich die tiefsten Tiefen seines Geistes. (Berdjajew 19)

Ebenso hebt der Psychologe Paul Vogel hervor: „Dostojewskij hat die Epilepsie auf seine Weise als sein Schicksal angenommen. Die Anfälle wurden ihm zu einem Element seines Lebens und seiner Lebensgeschichte und zu einer Quelle der Selbsterfahrung, die in seinem Werk einen einzigartigen Niederschlag fanden." (Vogel 33) Und Thomas Mann ergänzt: „Bei Dostojewski, dem Epileptiker, ist man beinahe gezwungen, in der Krankheit ein Produkt überschüssiger Kraft zu sehen, eine Explosion und einen Exzeß enormer Gesundheit, und sich von der Tatsache zu überzeugen, daß höchste Vitalität die Züge bleicher Bresthaftigkeit tragen kann." (Mann 2009, 55)

In den Romanen und Erzählungen von Dostoevskij leiden Murin in "Die Wirtin“ (1847) ${ }^{11}$, Nelly in den „Erniedrigten und Beleidigten“ $(1861)^{12}$, Fürst Myškin aus „Der Idiot“, Liza Trusockaja im „Ewigen Gatten“ $(1870)^{13}$, der Ingenieur Kirillov aus den „Dämonen“ und Smerdjakov aus den „Brüdern Karamasov“ (1880) ${ }^{14}$ an Epilepsie. Auch bei Raskolnikov $(1866)^{15}$ zeichnen sich Träume in krankhaften Zuständen durch ungewöhnliche Deutlichkeit, Klarheit und außerordentliche Ähnlichkeit mit der Wirklichkeit ab. (Vgl. Dostojewski 1984,77)

Der Welt der großen Romane Dostojevskijs liegt eine Metaphysik der Krankheit zugrunde. Das Sujet der Romane lässt sich nach dem Slavisten Andreas Guski auf eine einfache Formel bringen: etwas, das lange verschwiegen, verschleiert und verdrängt wurde, tritt schließlich zu Tage und wird offenbar. In allen Romanen wird die dynamische Spannung zwischen Verschweigen und Offenbaren erhöht durch die stark kriminalistische Anlage des Sujets. Der gleiche Mechanismus

\footnotetext{
${ }^{11}$ Chozjajka

${ }^{12}$ Unižennye i oskorblennye

${ }^{13}$ Večnyj muž

${ }^{14}$ Bratja Karamazovy

${ }^{15}$ Prestuplenie i nakazanie
} 
macht sich aber auch in der Rollen- und Verhaltenstypologie der Figuren bemerkbar: Hier stehen meist getarnte, verschlossene Protagonisten solchen gegenüber. die spontan und offen handeln und über Eigenschaften verfügen, sich im Nu alles von der Seele zu reden. Analog sind die Räume konstruiert: Die dunklen Winkel, Ecken, Dienstbotensteigen stehen den offenen, öffentlichen und lichtdurchflutenden Straßen, dem Ballsaal und der Brücke gegenüber. (Vgl. Guski 130)

Die Spuren der Epilepsie sind besonders im Roman „Die Dämonen“16 zu finden, dessen Entstehung auch mit der Verschlimmerung von Dostoevskijs Leiden zusammenfiel: Im Mittelpunkt des Romans stehen die reiche Gutsherrin Varvara Petrovna Stavrogina und ihr steter Begleiter Stepan Trofimovič Verchovenskij, ein Autor, dessen liberale, von ästhetischen Gesichtspunkten und ,erhabenen' moralischen Prinzipien bestimmte Einstellung auf überzogene Weise karikiert wird, und ihr rätselhaft agierender Sohn Nikolaj Vsevolodovič Stavrogin. In der Stadt hat sich ein Klub, der neue Ideen vertritt, gegründet, der von der Gattin des Gouverneurs unterstützt wird. Zentraler Akteur in ihm ist Stepan Trofimovič Verchovenskij, Die Entwicklung dieses Klubs zu den „Unsrigen“, die im Verlauf des Romans zu einer revolutionären Geheimgesellschaft mutieren, mündet schließlich in den Versuch, die bestehende Ordnung zu stürzen. Zum Kreis der Agierenden gehören Šatov und Kirillov. Der Ingenieur Kirillov, einen weltbürgerlichen Sozialismus vertretend, ist mager, immer gut gekleidet ,seine Sprache ist seltsam abgebrochen'. (Dostojewki 1986, 119) Sprachveränderungen kommen bei Epileptikern häufig vor, meint Dieter Janz. (Vgl. 192ff.) Kirillov liebt die Nacht, das Nachdenken und Tee. Er sagt, er könne aufwachen, wann er wolle. (Vgl. Dostojewski 148) So wird er von Janz als Aufwach-Epileptiker bezeichnet. (Vgl. Janz 471) Für ihn gibt es eine vollständige Freiheit erst,

\footnotetext{
${ }^{16}$ Im Januar 1870 liest Dostoevskij in Dresden in einer russischen Zeitung von der Ermordung des Studenten Ivanov durch einen gewissen Sergej Nečaev, den Anführer einer revolutionären Gruppe, von denen sich der Student getrennt hatte. Zur selben Zeit verbreitet sich in Russland die Nachricht von einer Aufstandsbewegung des bekannten Anarchisten Bakunin, die in ganz Russland Verschwörerzentralen unterhalten soll. Auch deutsche Zeitungen berichten diese Nachrichten. Anfang Oktober dieses Jahres entwirft Dostoevskij bereits das Ideengerüst zum künftigen Roman. Dostoevskijs Hass auf die „Progressisten“ und Nihilisten, die er in Genf aus nächster Nähe kennen gelernt hatte, als er 1867 und 68 dort lebte, war groß.
} 
wenn es einerlei sei, ob man lebe oder nicht, wer Schmerz und Angst besiegen könne, der werde Gott selbst. (Vgl. Dostojewski 149-151)

Kirillov wird kurz vor seinem Selbstmord von Šatov aufgesucht und dabei aus seinen fesselnden Gedanken gerissen. Auf Šatovs Hinweis, die Nächte nicht weiter zu durchwachen, antwortet er:

Es gibt Augenblicke, sie dauern nur fünf, sechs Sekunden, da spürt man plötzlich die Gegenwart ewiger Harmonie und hat sie völlig erlangt. Das ist nichts Irdisches; ich meine nicht, es sei etwas Jenseitiges, sondern nur, daß der Mensch in irdischer Gestalt nicht überleben kann. Man muß sich psychisch verwandeln oder sterben. (...) Währte es länger als fünf Minuten, die Seele ertrüge es nicht und müßte vergehen. In diesen fünf Sekunden durchlebe ich ein Leben und würde davon mein ganzes Leben hingeben, denn es lohnt. (...). Schatow fragt: Kirillow, überkommt Sie das häufig? Mal alle drei Tage, dann wieder einmal in der Woche. Leiden Sie an Fallsucht? Nein. Dann kommt sie noch. Seien Sie auf der Hut, Kirillow, ich hörte, gerade so beginnt die Fallsucht. Ein Epileptiker schilderte einmal ausführlich dieses ankündende Gefühl vor einem Anfall, haargenau wie Sie; fünf Sekunden nannte auch er und sagte, länger sei es nicht zu ertragen. Erinnern Sie sich an Mohammed und den Krug, aus dem nichts über den Rand floß, während er auf seinem Roß das Paradies durcheilte. Der Krug sind diese fünf Sekunden; es erinnerte gar zu sehr an Ihre Harmonie, und Mohammed war Epileptiker. Seien Sie auf der Hut; Kirillow, die Fallsucht! (Dostojewski 1985, Bd.3, 176f)

Sofort fallen die erwähnten Schilderungen des Dichters gegenüber Freunden auf: Glücksgefühl, Paradies, Mohammed.

Fürst Myškin, der Held aus dem Roman „Der Idiot“, wird mit 22 Jahren zur Behandlung seiner Epilepsie von seinem Vormund in die Schweiz geschickt. Als eine Besserung eingetreten ist, kehrt er nach Russland zurück, auch, um eine Erbschaftsangelegenheit zu klären. Fürst Myškin lernt nicht nur Rogožin kennen, einen späteren Rivalen, sondern seine entfernten Verwandten Epančin mit drei Töchtern, Nastas'ja Filipova, die lange von Tockij als Geliebte „gehalten“ und nun mit einem Sekretär des Generals Epančin verheiratet werden soll. Myškin mischt sich ein, um Nastas'ja zu retten, er will sie sogar heiraten, ebenso wie die Tochter des Generals Aglaja. Am Ende ersticht Rogožin, der auch Nastas'ja begehrt, diese. Am Totenbett verfällt Myškin in ein Delirium. Der Fürst wird in die Schweiz zurückgebracht und versinkt in epileptischer Demenz. 
Der Leser lernt den Fürsten in einem Abteil des Zuges nach St. Petersburg kennen: Dabei fallen seine „großen, ruhigen, blauen Augen auf, in deren Blick etwas Stilles, aber Bedrückendes lag, und die jenen seltsamen Ausdruck hatten, an welchem viele, vom ersten Moment an ein epileptisches Individuum erkennen." (Dostojewski 1986, 6)

Die Anfälle des Fürsten haben in der Kindheit mit fiebrig-nervösen Unruheempfindungen eingesetzt, dazu kommen Traurigkeit, Zerstreutheit, Kopfschmerzen und Vorahnungen, dass es zu einem Anfall kommen würde. Er erzählt den Epančins, dass seine häufigen Krankheitsanfälle ihn beinahe ganz zum Idioten gemacht hätten. (Vgl. 38)

Wenn die Anfälle zunehmen, verfällt er immer in eine Art Stumpfsinn, verliert gänzlich das Gedächtnis. Wie reagieren die Mitmenschen auf ihn? Erschrocken wenden sie sich ab, spotten, haben Mitleid, nennen ihn einen Idioten. (Vgl. 72, 475, 849) Myškin hat Verständnis für diese Reaktionen. Manche suchen seinen Rat, sein Mitgefühl, indem sie sich offenbaren. Aglaja meint, dass sein Hauptverstand besser sei als der vieler anderer Menschen. (Vgl. 110) Myškin wird mehrfach als Arzt bezeichnet (vgl. 146), weil er bspw. Terentevs schlechte Träume deuten kann:

Es wunderte mich sehr, daß der Fürst offenbar von meinen schlimmen Träumen weiß: Er sagte vorhin wörtlich, ich würde in Pawlowsk vielleicht weniger erregt sein und bessere Träume haben. Wie kommt er darauf? Entweder hat er medizinische Kenntnisse oder in der Tat einen ungewöhnlichen Verstand, der ihn vieles erraten läßt. (533)

Myškin erzählt der Familie des Generals Epančin von einem epileptischen Esel in Basel, der ihn von der russischen Melancholie erlöst, und von seiner Zeit in der Klinik im Wallis, die ihn aber nicht von seinen Anfällen wirklich hat heilen können. (Vgl. 78)

Die Ursachen und Begleiterscheinungen der epileptischen Erkrankungen sind vom Dichter als Natur und Kultur charakterisiert. Myškin führt selbst als Ursache seiner Erkrankung das Klima an. (Vgl. 76) Die epileptische Erkrankung des Fürsten verbindet sich mit sozialen, psychischen und vor allem geistigen Fähigkeiten. Diese zeigen sich auf folgenden Gebieten: Er kann schönschreiben. (Vgl. 45f.) Er durchschaut Menschen. Er kann in den Gesichtern der Menschen lesen. (Vgl. 94) Er wirkt wie ein Katalysator und Psychotherapeut. (Vgl. 114)

Für seinen Arzt Schneider in Wallis ist Myškin psychisch und intellektuell auf dem Niveau eines Kindes zurückgeblieben. Kinder bemerken Dinge, die andere nicht sehen. 
Myškins Verzicht auf die sinnliche Liebe lässt Rogožin in ihm einen armen Heiligen sehen. (Vgl. 20) Janz unterscheidet unter den Epileptikern Aufwach- und Schlaftypen, den Fürsten Myškin rechnet er zu den Aufwachtypen, weil diese sich meist erst auf einen äußeren Anstoß hin manifestieren und auch erst dadurch in Gang kommen, (...) daß sich derartige Anlässe wiederholen, und dagegen Schlaf-Epilepsien im allgemeinen ohne äußeren Anstoß einsetzen und weitgehend unabhängig von derartigen Anlässen verlaufen. (Vgl. Janz 1969, 462)

Zwei Anfälle werden im Romangeschehen geschildert, die im Sinne des deus ex machina wirken: Einmal errettet er Myškin vor dem Mordversuch Rogožins, und der zweite Anfall beendet die skandalträchtige Rede auf dem Verlobungsempfang der Epančins. Zwischen diesen Ereignissen erweist er sich als klug und hellsichtig, bisweilen kindlich, verwirrt, immer voller Mitleid: Bei einem Spaziergang erlebt Myškin die Vorboten eines Gewitters ${ }^{17}$ :

Er wußte es nicht, denn er fühlte sich den ganzen Tag in einem krankhaften Zustand wie seinerseits vor einem seiner Anfälle. (...) So dachte er unter anderem daran, daß es bei seinen epileptischen Zuständen unmittelbar vor einem Anfall (sofern dieser nicht im Schlaf eintrat) eine Phase gegeben hatte, in der nach tiefer Trübsal, seelischer Finsternis und Niedergeschlagenheit plötzlich für einige Augenblicke gleichsam sein Hirn aufloderte und alle Lebenskräfte sich in übermäßigem Tatendrang spannten. Das Gefühl für das Dasein und das eigene Ich steigerte sich in diesen Momenten, die aufzuckten wie ein Blitz, fast um das Zehnfache. Geist und Herz schienen von einem außergewöhnlichen Licht erleuchtet, (...). Wenn er in der fraglichen Sekunde, das heißt in dem letzten bei Bewußtsein erlebten Augenblick vor dem Anfall, sich mit klaren Verstand sagen konnte: Ja, für diesen Moment könnte man sein ganzes Leben hingeben! so war dieser allein für sich gewiß ein solches Opfer wert. Allerdings sah er sehr wohl auch die Dialektik seines Schlusses - zu deutlich standen ihm die geistige

17 „Der Anfall entwickelte sich also aus einer Krisensituation, deren Erregung lawinenartig anschwillt, und die schließlich zu einer Sprengung der Grenzen des Bewußtseins führt und ihre Energien im zuckenden Kampf des Leibes entlädt. (...) es war schwül, in der Ferne zog ein Gewitter auf, es wurde sehr schwül, die ersten Tropfen fallen, dann Gewitterregen (...) Diese Interjektionen artikulieren gewissermaßen die Vorgänge im Innern. Mit der Verflechtung des epileptischen Anfalls in den dramatischen Ablauf einer Situation erscheint der Anfall wie ein seelisch motivierter Ausbruch." (Vogel 34) 
Verkrümmung, das seelische Dunkel und die Idiotie als offenkundige Folge solchen „Hochgefühls“ vor Augen. (Dostojewski 1986, 309-311)

Dieses lange Zitat unterstreicht erneut die Parallelität zwischen Dostoevskijs Aussagen über den Verlauf eines epileptischen Anfalls und dieser Darstellung. Einschränkend muss hinzugefügt werden, dass der Dichter nicht eigenes Erleben im Detail nachzeichnet, sondern sein Interesse gilt auch den medizinischen Wissenschaften, dem Fortschritt beim Erkennen gerade von Nervenkrankheiten, von Persönlichkeitsspaltungen. Die Stadt St. Petersburg - entweder in glühender Sonne, siehe Beschreibung in "Schuld und Sühne" - oder in nebelhafter Feuchtigkeit, hatte kein gutes Klima.

Myškin beschreibt in der zitierten Textstelle das Vor-und Hochgefühl vor dem Anfall, den er dann im Gasthaus bei Rogožin erleidet: „Die Selbstreflexion des Fürsten Myschkin über seinen Zustand unmittelbar vor seinem Anfall zeichnet diesen als außerordentliches Glücksmoment, als einen fast mystischen Zustand, ja geradezu als ein Existenzial aus." (Sauder 10) Im Roman heißt es:

Ein epileptischer Anfall hatte ihn ereilt wie schon seit langem nicht mehr. Bekanntlich treten die Paroxysmen der Epilepsie oder Fallsucht in Bruchteilen von Sekunden ein. Das Antlitz des Kranken verzerrt sich plötzlich, er verdreht die Augen. Konvulsive Zuckungen und Krämpfe erfassen den ganzen Körper und sämtliche Gesichtsmuskeln. Dem voraus geht ein unvorstellbarer Schrei, dem nichts Menschliches mehr innezuwohnen scheint; ein Beobachter vermag sich nicht oder nur sehr schwer vorzustellen, daß ein Mensch ihn ausgestoßen hat. (...) Nicht wenige fühlen sich von dem Anblick eines Fallsüchtigen, dem in der Tat etwas Mystisches anhaftet, in Angst und Schrecken versetzt. Vermutlich bewirkte dieser Eindruck plötzlichen Entsetzens in der ohnehin grausigen Situation, daß Rogoschin auf der Stelle erstarrte und der Fürst somit vor dem Stoß des bereits auf inn gerichteten Messers bewahrt blieb. Unter heftigem Umschlagen und Schüttelkrämpfen glitt der Körper des Kranken die etwa fünfzehn Stufen hinab bis ans Ende der Treppe. Nach höchstens fünf Minuten entdeckte man ihn. (...) man kam darauf, daß es sich um einen Fallsüchtigen handelte. (Dostojewski 1986, 323)

Der Leser erhält hier einen genauen, fast sachlichen Bericht einer Diagnose, von der die Slavistin Renate Lachmann der Überzeugung ist, 
„dass die Selbstbeschreibungen, die Dostoevskij seine EpilepsieHelden äußern lässt, eine die objektivierende Psychoanalyse offenbar vorwegnehmende Bedeutung haben - so etwa Freuds Epilepsie-Analyse von 1928 am Beispiel der Brüder Karamasow. Wie die Hysterie erscheint demnach die Epilepsie als Hyperbel des Selbstausdrucks der agierenden Figuren, die der Erzähler im Modus der Beschreibung einsetzt, wobei das Verfahren der enargeia, der Voraugenführung, Dostoevskij nachhaltig zu gelingen scheint. (Lachmann, 133)

Im Roman „Die Brüder Karamazov“ kehren diese als Erwachsene in das Haus ihres Vaters zurück, gegenüber ihrem Vater, einem Lüstling und Narren, haben sie nur Verachtung und Hass übrig. Drei Brüder wünschen ihm den Tod. Als der Vater ermordet wird, fällt der Verdacht sofort auf den Sohn Dmitrij, sämtliche Indizien sprechen gegen ihn, er wird aber zu Zwangsarbeit in Sibirien verurteilt. Der tatsächliche Mörder ist der Epileptiker Smerdjakov (der Stinkende), ein illegitimer Sohn des alten Karamazov, der bei dessen Diener Grigorij aufwächst. Als ihm sein Ziehvater die biblische Schöpfungsgeschichte erzählt, weiß das Kind Smerdjakov zu antworten, dass Gott am ersten Tag seiner Schöpfung gar kein Licht gehabt habe, das erst am 4. Tag geschaffen worden sei. Grigorij gibt ihm eine Ohrfeige; nach einer Woche bekommt Smerdjakov den ersten epileptischen Anfall. (Vgl. Dostojewskij 1973 Bd.1, 181) Eine Heilung erscheint unmöglich. Der Leser erlebt ihn nur in einer kurzen Zeitspanne, in der sein Gesundheitszustand immer schlechter wird. Der schweigsame, menschenscheue, Tiere quälende Smerdjakov arbeitet als Koch im Haus des alten Karamazov und agiert unter der Maxime des zweiten Karamazov-Sohnes Ivan: Alles ist erlaubt. In einem Gespräch mit Ivan kündigt er für den nächsten Tag eine lange Epilepsie an. Er gibt zu, einen Anfall simulieren zu können: „Sicherlich kann man einen epileptischen Anfall nicht auf den Tag und die Stunde voraussagen, doch ein Vorgefühl kann man immer haben." (Bd.2, 352) Dieser wirft ihn nach dem Mord an Fëdor Karamazov auf das Krankenbett, und so kann er nicht als Täter bestimmt werden. Aus der Simulation fällt Smerdjakov dann in einen wirklichen Anfall. Bei Ivans letztem Besuch bei ihm sind seine Augen eingefallen, die unteren Lider blau. Smerdjakov wirkt wie ein Traum, wie ein Gespenst. (Vgl. 389) Nach dieser Begegnung begeht er Selbstmord, ohne den Mord an seinem Vater gestanden zu haben. Der sittliche Abstieg hat hier eine zunehmende Erkrankung zur Folge.

In allen, auch hier nicht dezidiert aufgeführten Beispielen stellt Dostoevskij die Epilepsie als reaktiv auf die Lebenssituation dar, während er genau weiß, dass seine Epilepsie genuin ist. Die Epilepsie in 
seiner Dichtung könnte auch eine Reaktion auf eine inakzeptable Wirklichkeit gewesen sein, wobei nur die Kenntnis der Epilepsie Dostoevskijs eine poetische Eindeutigkeit ganz verdecken könnte. Während alle sich mit Dostoevskijs Krankheit, allein durch seine Schriften, beschäftigenden Ärzte (und auch Schriftsteller) ganz eindeutig den Zusammenhang zwischen der Epilepsie und Genie konstatieren, zwischen Leben und Dichtung:

„(...) so sehr die Krankheit Dostojewskis Geisteskräfte bedrohte, sein Genie aufs engste mit ihr verbunden und von ihr gefärbt ist, daß seine psychologische Initiiertheit, sein Wissen um das Verbrechen und um das, was die Apokalypse "satanische Tiefen" nennt, vor allem seine Fähigkeit, geheimnisvolle Schuld zu suggerieren und sie den Hintergrund der Existenz seiner zum Teil entsetzlichen Geschöpfe bilden zu lassen, untrennbar mit ihr zusammenhängen. (Mann 2009, 49).

So gehen Literaturwissenschaftler nicht von dieser Eindeutigkeit aus. Der Slavist Klaus Städtke sieht Dostoevskijs Romane „stilistisch überaus komplex durchorganisiert, so dass direkte Rückschlüsse auf die Seelenverfassung des Verfassers nicht ohne weiteres zulässig seien." (Städtke 158) In der Folge spricht er von der Unberechenbarkeit, Labilität und Ekstatik der Menschen in Dostoevskijs Werk und davon, dass dessen Leben eigentlich in zwei Teile zerbrochen gewesen sei, in einen Teil, wofür er gelebt habe und in eine unbekannte Hälfte, in der alles fremd gewesen sei. (Vgl. 167) In einem Gespräch zwischen dem General und Myškin wird diese Aussage indirekt bestätigt: Der General bemerkt, „würde ein Romanschreiber es erzählen, wären es verworrene Phantastereien und Unwahrscheinlichkeiten." Und der Fürst berichtet von einem Mord: „(...) Hätte sich so was ein Literat ausgedacht - die Kenner des Volkslebens und die Kritiker hätten sofort geschrien, dergleichen sei ausgeschlossen." (Dostojewski 1986, 681) Auch Renate Lachmann ist der Auffassung, dass gerade Myškin seine Krankheit einer medizinischen Definition entzieht: „Myškins Epilepsie behält den Aspekt der heiligen Krankheit bei, der in ihrer vor-analytischen Phase eine Rolle gespielt hat. Myškin befindet sich quasi zwischen hyperbolischem Selbstausdruck und der Erfahrung des Erhabenen." (Lachmann 134)

Myškin könnte Dostoevskijs Medium geworden sein; im Roman wird das Leben des Dichters transzendiert.

Der Germanist Gerhard Sauder hat erarbeitet, dass ,die „Entlastung' und Aufwertung des Kranken als Tendenz der Moderne sich mit zahlreichen literarischen Texten über Epilepsie belegen lasse. Das 
Paradigma dafür hat Dostojewskij in seinem Roman Der Idiot geschaffen. (Sauder 10)

Dabei fällt ins Auge, dass der nicht medizinische Zugang zum Werk des Dichters die durch die Krankheit entstandene Poesie hervorhebt und einen ganzheitlichen Charakter aufweist, während die medizinische Wissenschaft den Zusammenhang zwischen Krankheit und einer besonderen kreativen Fähigkeit ,beschwört'. Allerdings sind Leben und Werk Dostoevskijs ohne die Erhellung und das Erleben des Krankseins nicht vollständig zu erschließen. Letztendlich erlebte er seine Krankheit als Fluch und Gnade zugleich.

\section{Literatur}

Berdjajew, Nikolaj. Die Weltanschauung Dostojewskijs. München: Beck,1925

Catteau, Jacques. Dostoevsky and the process of literary creation. Cambridge: Unv. Press, 1989

Dostojewskaja, Anna G. Erinnerungen. Berlin: Rütten\&Loening, 1976

Dieselbe. Tagebuch. Königstein/Ts: Athenäum, 1985

Dostojewskaja, Ljubov'. Dostojewski. München: Reinhardt, 1923

Dostojewski, Fjodor M. Raskolnikow. Berlin: Aufbau, 1956 (Übersetzung von Hermann Röhl)

Derselbe. Tagebuch eines Schriftstellers. München: Piper, 1972

Derselbe. Die Brüder Karamasow. 2 Bd..Leipzig: Reclam, 1973 (Übersetzung von Hermann Röhl)

Dostojewski, Fjodor- Dostojewskaja; Anna: Briefwechsel 1866-1880. Berlin: Rütten\&Loenig, 1982

Derselbe. Briefe in zwei Bänden, hrsg. von Ralf Schröder. Leipzig: Insel, 1984

Derselbe. Die Dämonen. 2 Bd. Berlin: Aufbau, 1985 (Übersetzung von Günter Dalitz)

Derselbe. Der Idiot. Berlin: Aufbau, 1986. (Übersetzung von Hartmut Herboth)

Derselbe. Gesammelte Briefe 1833-1881. München / Zürich: Piper, 1986.

Engelhardt, Dieter von: „Epilepsie im Werk Dostjewskijs“. DostoevskyStudies, vol. V, 2007, 25-40.

Freud, Sigmund. Dostojewski und die Vatertötung. Gesammelte Werke. Bd. 14, Frankfurt a. M: Fischer, 1972, 399-418

Fülöp-Miller, Rene. „Die Krisis in Dostojewskis Leben. Eine Darstellung auf Grund des bisher unbekannt gebliebenen Nachlasses". Dostojewski am Roulette, hrsg. von Rene Fülöp-Miller und Friedrich Eckstein. München: R. Piper\&Co, 1925, X- LXXXVII 
Fung, Paul. Dostoevsky and the Epileptic Mode of Being. London: Legenda, 2015

Guski, Andreas. „Verbergen und Offenbaren bei Dostoevskij“. Schweizerische Beiträge zum IX. Internationalen Slavistenkongress in Bratislava. Bern 1993 (=Slavica Helvetica, Bd. 42), 113-125

Janz, Dieter. "Leitbilder der Epilepsie bei Hippokrates und Paracelsus". Jahrbuch für Psychologie. Psychotherapie und Medizinische Anthropologie, hrsg. von Victor E. Freiherr von Gebsattel, P. Christin, W. J. Revers und T. Tellenbach. 14. Jahrgang Freiburg / München: Karl Alber, 1966, 2-16

Derselbe. Epilepsien. Stuttgart: Thieme, 1969

Jung, Carl. Analytische Psychologie und Erziehung. Heidelberg: Niels Kampmann, 1924

Kowalewskaja, Sofja W. Jugenderinnerungen. Bremen: Europäischer Literatur, 2010

Lachmann, Renate. „Die Lehre der Affekte und ihre Rolle im Werk Dostoevskijs“, arcadia, internationale Zeitschrift für Literatur, hrsg. von Vivian Liska, John Neubauer und Jürgen Weitheimer, Bd. 44, Berlin, New York, De Gruyter, 2009, 121-136

Ljaskovskaja, Ol'ga A.: V.G.Perov. Osobennosti tvorčeskogo puti chudožnika. Moskva: Iskusstvo, 1979

Mann, Thomas. „Russische Dichtergalerie“. Essays II 1914-1926 Frankfurt a.M.: Fischer 2002, hrsg. von Hermann Kurzke. Große kommentierte Frankfurter Ausgabe, von Heinrich Detering, Eckhard Heftrich, Hermann Kurzke. Bd. 15.1, 579-581

Derselbe. „Dostojewski - mit Maßen“. Essays VI 1945-1950. Frankfurt a.M.: Fischer, 2009, hrsg. von Herbert Lehnert. Große kommentierte Frankfurter Ausgabe, hrsg. von Heinrich Detering, Eckhard Heftrich, Hermann Kurzke. Bd. 19.1, 42-62

Mereschkowski, Dmitri. S. Tolstoi und Dostojewski. Leben, Schaffen, Religion. München: Voegels, 1924

Merten, Sabine: Die Entstehung des Realismus aus der Poetik der Medizin. Wiesbaden: Harrassowitz, 2003

Panaeva, Avdot'ja. Vospominanija. Moskva: Zacharov, 2002

Rattner, Joseph. „Miteinander leben lernen“. Zeitschrift für Tiefenpsychologie, Persönlichkeitsbildung und Kulturforschung. Heft 1. Berlin 1993, 43; zitiert nach: Riester, Jutta. Die Menschen Dostojewskis. Göttingen: V\&R unipress, 2012, 158

Sauder, Gerhard. "Sinn und Bedeutung von Krankheitsmotiven in der Literatur". Das ist eine alte Krankheit. Epilepsie in der Literatur. Hrsg. von Dietrich v. Engelhardt, Dietrich, Hansjörg Schneble, Peter Wolf. Stuttgart; Schattauer, 2000, 1-11 
Schult, Maike. „Fascinosum et tremendum. Dostoevskijs Schaffen im Porträtvergleich". Jahrbuch der Deutschen DostojewskijGesellschaft 19 (2012), 109-121

Solov'ev', Vsevolod S. Chronika četyrëch pokolenij iz Vospominanij o Dostoevskom. St. Petersburg 1904, ohne Angabe des Verlages, 3161

Städtke, Klaus: Dostojewski für Eilige. Berlin: Aufbau, 2004

Vogel, Paul. „Von der Selbstwahrnehmung der Epilepsie. Der Fall Dostojewskij". Jahrbuch für Psychologie. Psychotherapie und Medizinische Anthropologie, hrsg. von Victor E. Freiherr von Gebsattel, P. Christin, W. J. Revers und T. Tellenbach. 14. Jahrgang Freiburg/ München: Karl Alber, 1966, 30-37

Zweig, Stefan. „Drei Meister. Dostojewski“. Essays. Auswahl 1907-1924. Leipzig: Anton Kippenberg, 1983, 369-397

\section{FYODOR M. DOSTOEVSKY'S LIFE'S WORK IN THE CONTEXT OF EPILEPSY}

Medical doctors and friends alike have confirmed that Dostoevsky suffered from epilepsy. It was an essential part of the poet's life, his selfdetermination and literary creativity. Thus, he was able to appreciate all the ups and downs of human life, for example, in the character and ambivalence of Prince Myshkin. The novel became a paradigm of modernism in which medicine and literature are no longer contradictions, but offer an analytic view of human existence.

Keywords: Dostoevsky's portrait of life, epilepsy: sacred disease, curse, fear, or transcendence, Prince Myshkin 